\title{
Active and Reactive Power Metering in Non-Sinusoidal Conditions Using Newton Type Algorithm
}

\author{
VladimirTerzija $^{1}$, Vladimir Stanojevic ${ }^{2}$, Zoran Lazarevic ${ }^{2}$, Marjan Popov $^{3}$, \\ ${ }^{1}$ ABB Calor Emag Mittelspannung GmbH, \\ Ratingen, Germany, e-mail: Vladimir.Terzija@de.abb.com \\ ${ }^{2}$ Faculty of Electrical Engineering, \\ Belgrade, Ser\&Mo, e-mail: lazarevic@kiklop.etf.bg.ac.yu; vstanojevic@web.de \\ ${ }^{3}$ Delft University of Technology, Power Systems Laboratory, \\ Delft, The Netherlands, e-mail: M.Popov@ieee.org
}

\begin{abstract}
This paper presents a new two stage Newton Type numerical algorithm for active and reactive power measurement in electric power systems with sinusoidal, non-sinusoidal linear and non-sinusoidal nonlinear circuits. In order to estimate their spectra, in the first stage of the algorithm the current and voltage signals are separately processed, whereas in the second stage the power components are calculated. As the main advantage, this technique provides measurements not sensitive to frequency deviations. The algorithm performance is tested using computer simulated tests.
\end{abstract}

Keywords: power systems, power measurement, nonlinear estimation, computer simulation, laboratory testing.

\section{Introduction}

Harmonic pollution is of raising presence in the contemporary power systems. The main cause of this are large non-linear elements such as power electronics used for the system operation (e.g. thiristor controlled compensers or reactors), non-linear loads such as the arc furnaces, or high power converters. There is a great number of papers concerned with development of measurement techniques and algorithms for electric power systems with non-sinusoidal linear and non-linear circuits.

However, many of those numerical algorithms are sensitive to frequency variations, for example, DFT (Discrete Fourier Transformation) or LSE algorithm (Least
Squares Error). The frequency deviation occurs after a large block of load or a large generator unit is connected to or disconnected from the power system. Frequency variations are much more likely to occur for the loads supplied by a generator isolated from the utility system.

Numerical algorithm presented in this paper considers the system frequency as an unknown parameter of the model to be estimated and in this way solves the problem of sensitivity to frequency variations in wide range. With the introduction of the power frequency in the vector of the unknown model parameters, the model itself becomes nonlinear and the strategies of nonlinear estimation must be used. For the purpose of the voltage and current spectra estimation, the Newton Type Algorithm (NTA) is developed and successfully implemented in the active and reactive power measurement. The NTA can be also applied in determining some other quantities, for example, those defining electric power quality or in the procedure of identifying harmonic sources in the power system.

\section{NTA Algorithm Development}

Let us assume the following observation model of the input voltage (or current) signal, digitized at the measurement device location:

$$
v(t)=h(\mathbf{x}, t)+\xi(t)
$$


in which $v(t)$ is an instantaneous voltage at time $t, \xi(t)$ a zero mean random noise, $\mathbf{x}$ a suitable parameter vector and $h(\cdot)$ nonlinear function expressed as:

$$
h(\mathbf{x}, t)=V_{0}+\sum_{k=1}^{M} V_{k} \sin \left(k \omega t+\varphi_{k}\right)
$$

For the generic model (2), a suitable vector of unknown parameters is given by:

$$
\mathbf{x}=\left[V_{0}, \omega, V_{1}, \ldots, V_{M}, \varphi_{1}, \ldots, \varphi_{M}\right]^{T}
$$

where $V_{0}$ is the magnitude of the decaying DC component at $t=0, M$ is the highest order of the harmonics presented in the signal, $\omega$ is the fundamental angular velocity, equal to $2 \pi f, f$ being frequency, $V_{k}$ is the magnitude of the $k$-th harmonic and $\varphi_{k}$ is the phase angle of the $k$-th harmonics $(k$ $=1, \ldots, M)$. The equivalent signal model can be assumed for the current signal. The number of unknowns, i.e. the model order, is $n=2 M+2$.

The input signals are sampled during a finite period of time, called data window. Taking $N$ samples to a data window, a set of $N(N \geq n=2 M+2)$ nonlinear equations, given by (1) and (2), in $n$ unknowns are obtained. The problem is to solve the overdetermined system of nonlinear equations, i.e. to determine the unknown model parameters.

The key relation of the NTA algorithm is given by:

$$
\hat{\mathbf{x}}_{i+1}=\hat{\mathbf{x}}_{i}+\left(\mathbf{J}_{i}^{T} \mathbf{J}_{i}\right)^{-1} \mathbf{J}_{i}^{T}\left(\mathbf{v}-\mathbf{h}\left(\hat{\mathbf{x}}_{i}, t\right)\right)
$$

where $i$ is an iteration index, $\hat{\mathbf{x}}$ is estimated vector, $\mathbf{J}_{i}^{\#}=\left(\mathbf{J}_{i}^{T} \mathbf{J}_{i}\right)^{-1} \mathbf{J}_{i}^{T}$ is referred to as a left pseudoinverse of Jacobian $\mathbf{J}_{i}, \mathbf{v}$ is an $(N \times 1)$ measurement vector, $\mathbf{h}\left(\hat{\mathbf{x}}_{i}, t\right)$ is an $(N \times 1)$ vector of nonlinear functions determined by the assumed mathematical model of the input signal and $N$ is the number of samples belonging to the data window.

Jacobian matrix $\mathbf{J}$ is an $(N \times n)$ matrix, having as its elements the partial derivatives of the signal model (2). If $\mathbf{j}$ is an arbitrary row of the Jacobian, then

$$
\begin{aligned}
& \mathbf{j}=\left[j_{1}, j_{2}, j_{3}, \cdots, j_{2+2 M}\right] \\
& j_{1}=\frac{\partial h(\mathbf{x})}{\partial V_{0}}=1 \\
& j_{2}=\frac{\partial h(\mathbf{x})}{\partial \omega}=\sum_{k=1}^{M} V_{k} k t \cos \left(k \omega t+\varphi_{k}\right)
\end{aligned}
$$

$$
\begin{aligned}
& j_{2+k}=\frac{\partial h(\mathbf{x})}{\partial V_{k}}=\sin \left(k \omega t+\varphi_{k}\right) \\
& j_{2+M+k}=\frac{\partial h(\mathbf{x})}{\partial \varphi_{k}}=V_{k} \cos \left(k \omega t+\varphi_{k}\right)
\end{aligned}
$$

where $k=1, \ldots, M$.

Equation (4) is derived first by linearization, i.e. by Taylor series expansion of (1) and neglecting higher order terms, and then by applying Least Square Error estimation method.

\section{Two Stage Numerical Algorithm}

Block diagram of the two stage numerical algorithm is presented in Figure 1.

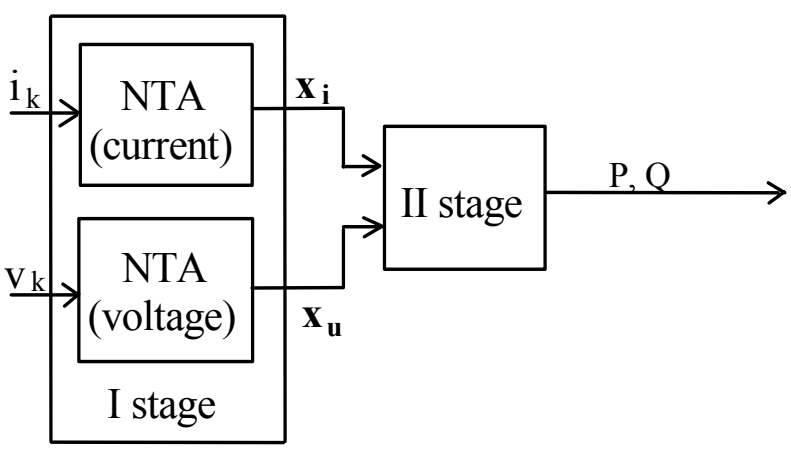

Figure 1: Block diagram of the two stage NTA algorithm.

In Figure $1, i_{k}$ and $v_{k}$ are current and voltage samples, respectively. The inputs to the second stage of the algorithm are the voltage and current signals spectra, contained in the vectors $\mathbf{x}_{\mathbf{i}}$ and $\mathbf{x}_{\mathbf{u}}$, estimated in the first stage of the algorithm. In the second stage of the algorithm, active and reactive powers are calculated. The average power is expressed as:

$$
P_{a v}=V_{0} I_{0}+\sum_{k=1}^{M} \frac{1}{2} V_{k} I_{k} \cos \left(\varphi_{k}-\psi_{k}\right)
$$

where $\varphi_{k}$ and $\psi_{k}$ are voltage and current $k$-th harmonics phase angles.

There is no unique definition for the reactive power under non-sinusoidal conditions. In this paper Budeanu's reactive power definition [1] is used:

$$
Q_{B u d}=\sum_{k=1}^{M} \frac{1}{2} V_{k} I_{k} \sin \left(\varphi_{k}-\psi_{k}\right)
$$




\section{Algorithm Testing}

The proposed numerical measurement algorithm is tested through computer simulated tests, as follows:

1. dynamic test, and

2. test using dynamic simulation of power system.

\section{A. Dynamic test}

Dynamic test consists of processing the following computer generated distorted voltage and current signals:

$$
\begin{aligned}
v(t)= & 100 \cos (\omega t+45)+50 \cos (3 \omega t+120) \\
& +30 \cos (5 \omega t+150)+20 \cos (7 \omega t+280)[\mathrm{V}] \\
i(t)= & 100 \cos (\omega t)+40 \cos (3 \omega t+60) \\
+ & 20 \cos (5 \omega t+30)+10 \cos (7 \omega t+130)
\end{aligned}
$$

At first, from 0 to $0.03 \mathrm{~s}$, pure sinusoidal waves of voltage and current were generated, with magnitude of 100 $\mathrm{V}(\mathrm{A})$ and $50 \mathrm{~Hz}$. At $\mathrm{t}=0.03 \mathrm{~s}$ the magnitude step change of $10 \%$ occurred. To approve the NTA algorithm insensitivity to frequency deviations, the frequency was also step changed at $0.03 \mathrm{~s}$ from $50 \mathrm{~Hz}$ to $49 \mathrm{~Hz}$, and then sinusoidal changed with time:

$$
f(t)=49+2 \sin (2 \pi \cdot 1.2 t)
$$

with frequency of $1.2 \mathrm{~Hz}$ (see Figure 4) which is approximately the natural frequency of synchronous generators swinging in a power system. Note that such a large frequency deviations are not likely to occur in a real power system due to inertia of generators' rotors. In this paper such large frequency variations are intentionally imposed to the voltage and current signals in order to investigate the robustness of the algorithm.

Generated voltage and current are presented in Figures 2 and 3. Real and estimated frequency is presented in Figure 4, while estimated power components are shown in Figure 5.

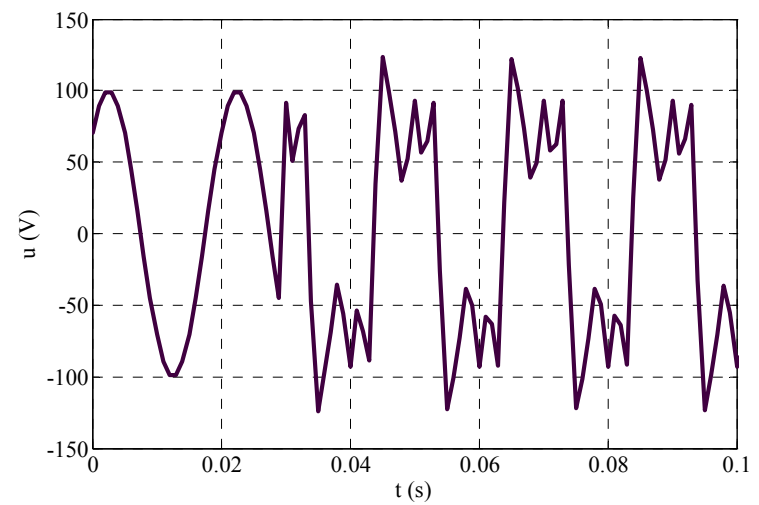

Figure 2: First five periods of computer generated voltage.

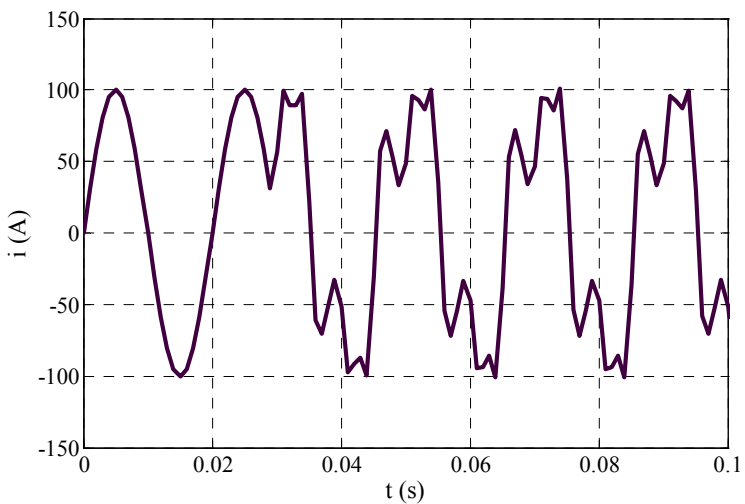

Figure 3: First five periods of computer generated current.

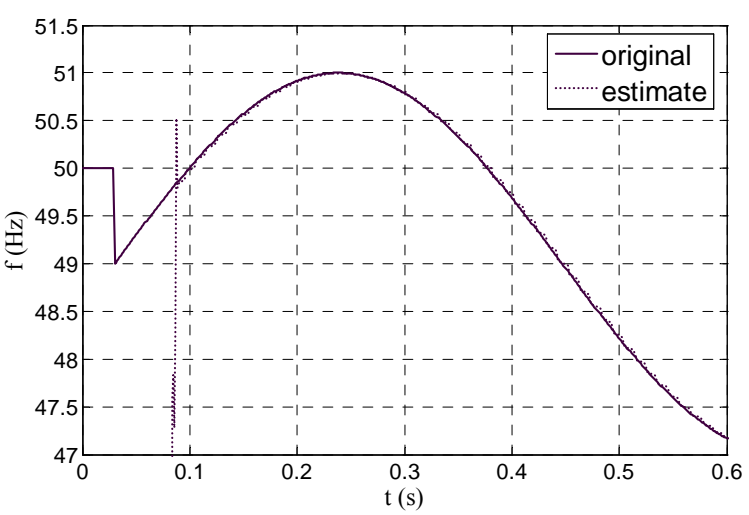

Figure 4: Original and estimated voltage frequency.

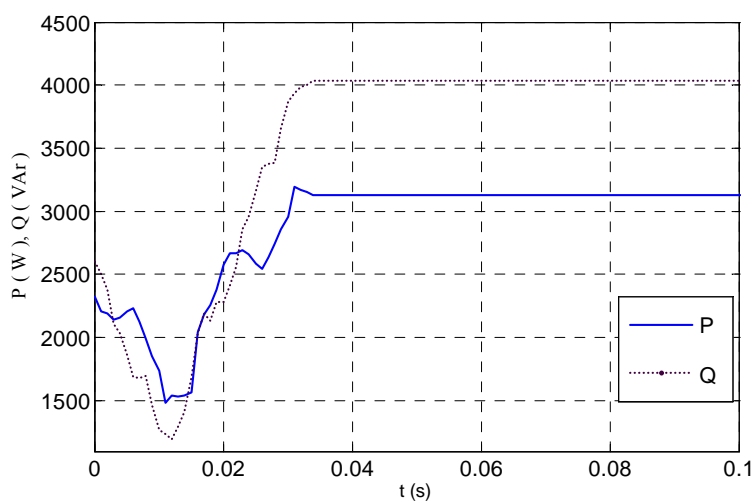

Figure 5: Power components estimated by NTA.

From Figure 5 it could be noticed that after a short period of convergence, the proper results for active and reactive power are obtained by the NTA algorithm: $3127 \mathrm{~W}$ and 4039 VAr.

On the contrary, the results obtained by using the Fast Fourier Technique are incorrect due to the "leakage" effect of the FFT in case when the assumed frequency $(50 \mathrm{~Hz})$ differs from the actual one. These results are shown in Figure 6. 


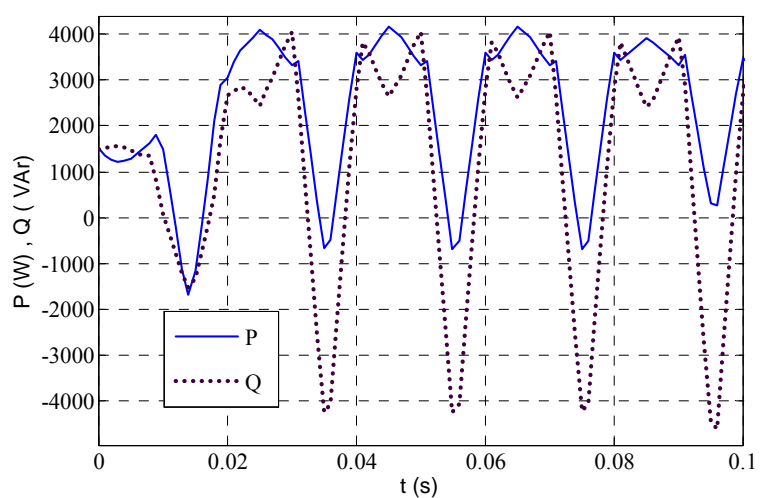

Figure 6: Power components estimated by the FFT algorithm

\section{B. Power system dynamic simulation}

For this purpose, an Electromagnetic Transient Program (EMTP) simulation code has been developed at TU Kaiserslautern (Germany). Through the EMTP simulation, the pure sinusoidal and distorted voltage and current signals are generated. In Figure 7 a single-line diagram of $220 \mathrm{kV}, 50 \mathrm{~Hz}$ test power transmission system is presented. In the same figure the power system parameters are given.

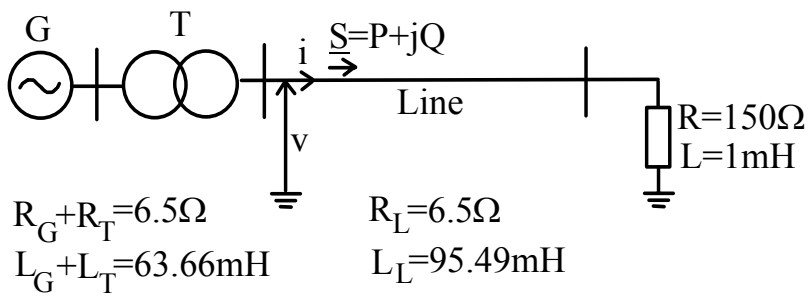

Figure 7: Test power system.

In this test, the fundamental harmonic of the electromotive force $(E=326.6 / \sqrt{2} \mathrm{kV}, 100 \%)$ the third $(31.41 \%)$ and the fifth $(17.15 \%)$ harmonics are superimposed. The generated higher order harmonics caused the distortion in the measured voltage $v(t)$ and current $i(t)$. Both the simulated voltage $v(t)$ and the current $i(t)$ are depicted in Figures 8 and 9, respectively.

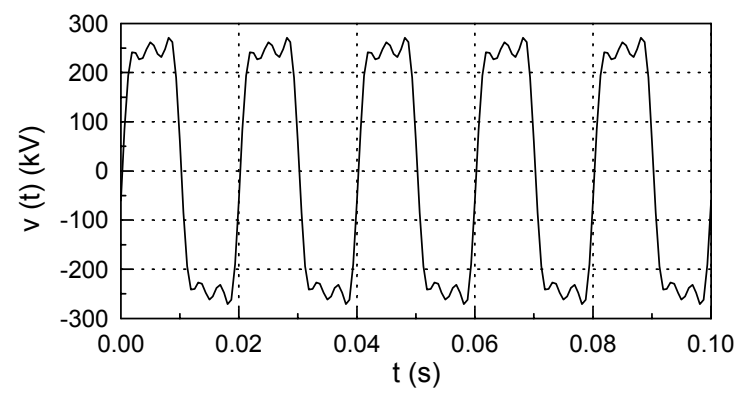

Figure 8: Input voltage signal.

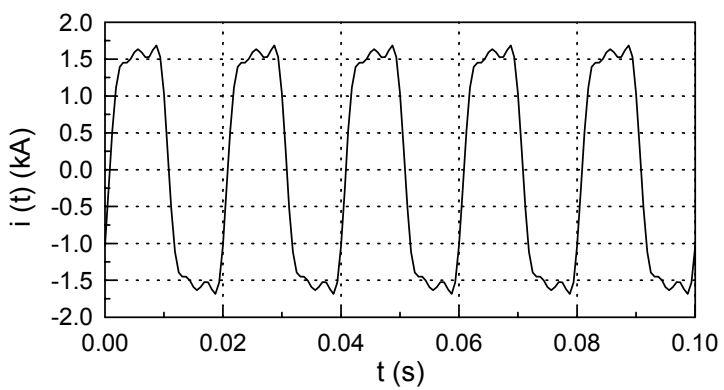

Figure 9: Input current signal.

By applying the presented two stage NTA algorithm, the active and reactive powers depicted in Figure 10 are estimated. The high accuracy is obtained. The relative errors were less than $10^{-4} \%$.

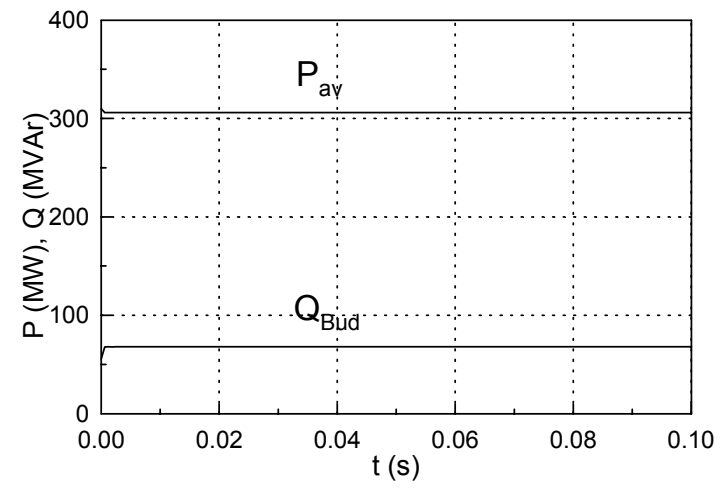

Figure 10: Estimated power components (dynamic simulation of power system).

\section{Conclusion}

In this paper a new numerical measurement algorithm for digital metering of power is presented and tested. It is based on the application of the Newton Type Algorithm, a nonlinear estimator suitable for the spectrum estimation of distorted input signals. The algorithm is not sensitive to the frequency changes of the input signal, which are typical for power systems during faults and abnormal operations, or for the electrical machines with the variable speed. The algorithm is tested through dynamic computer simulated tests, as well as by using data obtained from dynamic simulation of power system operated under non-sinusoidal conditions. The obtained results confirmed a high accuracy of the developed algorithm. The fast algorithm convergence offers the opportunity to apply the algorithm in processes with fast/very fast transients. Beside active and reactive power metering, this algorithm also enables measuring and estimation of other power quality quantities, such as: THD, apparent power, distortion power, power factor, RMS. The technique is not limited to the measurement applications in power systems only. It may be used in designing the algorithms for some other and new applications in the other field of engineering, as well. 


\section{REFERENCES}

[1] C.I.Budeanu, "Puissances Reactives at Fictives", Institut Romain de l'Energie, Publ. 2, Bucharest, Romania, 1927.

[2] S.Fryze, "Active, Reactive and Apparent Power in Nonsinusoidal Systems", Przegled Elektrotek. No 7, pp. 193-203, 1931.

[3] H.L.Kusters, W.J.M.Moore, "On the Definition of Reactive Power Under Non-Sinusoidal Conditions", IEEE Trans. on Power Apparatus and Systems, Vol. PAS-99, pp. 1845-1854, Oct. 1980.

[4] A.Emanuel (Chairman) et al., „Practical Definitions for Powers in Systems with Nonsinusoidal Waveforms and Unbalanced Loads: A Discussion“, IEEE Trans. on Power Delivery, Vol. 11, No. 1, pp. 79-101, January 1996.

[5] A.Routray, A.K.Pradhan, K.P.Rao, „A novel Kalman filter for frequency estimation of distorted signals in power systems", IEEE Trans. on Instrumentation \& Measurements, Vol. 51, Issue 3, pp. 469-479, June 2002.

[6] R.Arsenau, P.S.Filipski, "Effects of Harmonics on Warrhour and Demand Meters", CEA Meeting, Calgary, Oct. 1985.

[7] J.J.Hill, W.E.Alderson, "Design of a Microprocessor Based Digital Wattmeter", IEEE Trans. on Industrial Electronics and Control Instrumentation, Vol. IECI-28, No. 3, pp. 180-184, Aug. 1981

[8] M.Kezunovic et al. "New Approach to the Design of Digital Algorithms for Electric Power Measurement", IEEE Trans. on Power Delivery, Vol. 6, No. 2, April 1991.

[9] G.Bucci, C.Landi, "On-Line Digital Measurement for the Quality Analysis of Power Systems Under Non-Sinusoidal Conditions"' Proc. IEEE Instr. and Measurements Tech. Conference, Brusseles, Belgium, pp. 934-938, June 1996.

[10] V.Terzija et al., "Voltage Phasor and Local System Frequency Estimation Using Newton Type Algorithm", IEEE Trans. on Power Delivery, Vol. 9, No. 3, pp. 1368-1374, July 1994.

[11] V.Terzija, M.Djuric, "Direct Estimation of Voltage Phasor, Frequency and its Rate of Change Using Newton's Iterative Method", Electrical Power \& Energy Systems, Vol. 16, No. 6, pp. 423-428, 1994.

[12] V. Terzija, D. Nelles, "Parametrische Modelle des Lichtbogens und Parameterschätzung auf Grund der simulierten und echten Daten," TB 183/93, Univ. Kaiserslautern, Kaiserslautern, Germany, July 1993. 